ORIGINAL

\title{
Evaluation of the physicochemical and functional properties of Colombian bee pollen
}

\section{Evaluación de las propiedades fisicoquímicas y funcionales del polen apícola colombiano}

\author{
Carlos Fuenmayor B, ${ }^{1,2}$ Ph.D, Carlos Zuluaga D, ${ }^{1}$ M.Sc, Consuelo Díaz M, ${ }^{1}$ Ph.D, \\ Marta Quicazán de C, ${ }^{1 *}$ Ph.D, María Cosio, ${ }^{2}$ Ph.D, Saverio Mannino, ${ }^{2}$ Ph.D.
}

\begin{abstract}
${ }^{1}$ Universidad Nacional de Colombia, Instituto de Ciencia y Tecnología de Alimentos - ICTA, Ciudad Universitaria, Av. Cra. 30 \# 45-03 Ed. 500C, 111321. Bogotá, Colombia. 'Universidad de Milán, Departamento de Ciencias Alimentarias, Ambientales y Nutricionales, Via Mangiagalli 25, 20133 Milán, Italia. *Correspondencia: mcquicazand@unal.edu.co
\end{abstract}

Received: February 2013; Accepted: September 2013.

\begin{abstract}
Objective. To establish current knowledge about Colombian bee-pollen from a point of view nutritional and functional, contributing towards creating national technical standards and the identification of chemical differentiation factors for further researches. Material and methods. One hundred ninetysix samples of dried bee pollen were collected in the center region of Colombia known as Cundiboyacense high plateau, where nearly $90 \%$ of total bee pollen production is concentrated in this country. Performed physicochemical analyses in this study were: moisture, $\mathrm{pH}$, acidity, ash, carbohydrates, lipids, proteins, dietary fiber, fatty acid profile and mineral elements. Results. Bee pollen from this region had $7.7 \pm 5.2 \mathrm{~g} / 100 \mathrm{~g}$ moisture content, and a following centesimal composition based on dry matter: ashes $2.5 \pm 0.4 \mathrm{~g}$; lipids $6.90 \pm 3.5 \mathrm{~g}$; proteins $23.8 \pm 3.2 \mathrm{~g}$ and total dietary fiber $14.5 \pm 3.5 \mathrm{~g}$. The most abundant fatty acids were a-linolenic, palmitic and linoleic. Carbohydrates were the main components and fructose and glucose the most concentrated sugars. The predominant minerals assessed here were potassium, calcium and magnesium. The results were also discussed in terms of the characteristics found in Colombian bee-pollen in comparison to international regulations and findings for other varieties of commercial bee-pollen from eight different countries. Conclusions. The results found in this study suggest that bee-pollen may be used as a dietary supplement and agree with bibliographical reports and international regulations. Such characterization will enable to be proposed technical standards in line with Colombian bee-pollen properties and it is expected to improve marketing and production chain conditions.
\end{abstract}

Key words: Apis mellifera, dietary supplements food composition, food quality, physicochemical analysis (Source: DeCS). 


\section{RESUMEN}

Objetivos. Generar conocimiento actualizado sobre el polen colombiano desde un punto de vista nutricional y funcional, contribuyendo a la creación de estándares técnicos nacionales y la identificación de factores químicos de diferenciación para futuras investigaciones. Materiales y métodos. Ciento noventa y seis muestras de polen seco fueron recolectadas en la región central de Colombia conocida como el altiplano cundiboyacense, donde se concentra cerca del $90 \%$ de la producción total en este país. Los análisis fisicoquímicos realizados en este estudio fueron: humedad, pH, acidez, cenizas, carbohidratos, lípidos, proteínas, fibra dietaria, perfil de ácidos grasos y minerales. Resultados. El polen de esta región tuvo un contenido de humedad de $7.7 \pm 5.2 \mathrm{~g} / 100 \mathrm{~g}$ y una composición centesimal basada en materia seca: cenizas $2.5 \pm 0.4 \mathrm{~g}$; lípidos $6.90 \pm 3.5 \mathrm{~g}$; proteínas $23.8 \pm 3.2 \mathrm{~g}$ y fibra dietaria total $14.5 \pm 3.5 \mathrm{~g}$. Los ácidos grasos más abundantes fueron a-linolénico, palmítico y linoleico. Los carbohidratos fueron los componentes principales con fructosa y glucosa siendo los azúcares más concentrados. Los minerales predominantes evaluados fueron potasio, calcio y magnesio. Los resultados también fueron discutidos en términos de las características encontradas en el polen colombiano en comparación a normatividades internacionales y hallazgos para otras variedades de polen comercial de ocho países diferentes. Conclusiones. Los resultados encontrados en este estudio sugieren que el polen apícola puede ser utilizado como suplemento dietario y es coherente con los reportes bibliográficos y normativas internacionales. Tal caracterización permitirá proponer estándares técnicos de acuerdo a las propiedades del polen colombiano y se espera que se mejore la comercialización y las condiciones de la cadena de producción.

Palabras clave: Apis mellifera, análisis fisicoquímico, composición de alimentos y suplementos dietarios, calidad de alimentos (Fuente: DeCS).

\section{INTRODUCCIÓN}

El polen de abeja, también conocido como polen apícola, polen corbicular o polen de abeja recogido, es la principal fuente de proteínas para las colonias de abejas. Las abejas obreras transportan este recurso desde las flores a la colmena sobre sus patas traseras como gránulos que forman con los movimientos de sus patas delanteras usando peines, pelos y secreciones salivares (1). Una vez en la colmena, las abejas almacenan el polen en celdas donde se somete a procesos similares al ensilado, antes de ser consumido por crías y adultos; luego es conocido como pan de abeja (2). El manejo adecuado de la colmena promueve la recolección adicional de polen dirigido a su comercialización para el consumo humano, ya que puede ser considerado como alimento o un aditivo alimentario debido a su contenido de una amplia gama de nutrientes $(1,3-5)$. También representa un complemento dietario funcional, especialmente debido a sus capacidades antioxidantes y antimicrobianas, su composición de micronutrientes, su perfil de ácidos grasos y sus funciones terapéuticas o preventivas de enfermedades (6-9).

Los apicultores ubican trampas en la entrada de la colmena a través de las cuales las abejas obreras no pueden pasar fácilmente con ambas patas gránulos de polen y se ven obligadas a soltarlos en bandejas que son retiradas periódicamente y de las cuales se remueven los gránulos de polen y son sometidos a un proceso posterior para prolongar su vida útil,

\section{INTRODUCTION}

Bee pollen, also regarded, as apicultural pollen, corbicular pollen, or bee-collected pollen, is the main source of protein for bee colonies. Worker bees transport this resource from flowers into the hive by carrying it on their hind legs as pellets that they form with movements of their front legs using combs, hairs and salivary secretions (1). Once in the hive, bees store pollen in cells where it undergoes processes similar to ensiling, prior to being eaten by pups and adults; it is then referred to as bee bread (2). Proper hive management promotes additional pollen collection aimed at marketing it for human consumption, since it can be considered as food or food additive due to its content of a wide range of nutrients $(1,3-5)$. It also represents a functional dietary supplement, especially because of its antioxidant and antimicrobial activities, its micronutrients composition, its fatty acid profile and its therapeutic or diseasepreventing functions (6-9).

Beekeepers thus locate traps in the hive entrance through which the worker bees cannot pass easily with both legs carrying pollen pellet so they are forced to drop them onto trays which are periodically withdrawn and from which pollen pellets are removed and subjected to further processing for prolonging shelf-life, usually drying processes. 
generalmente mediante procesos de secado.

La comercialización y consumo actual de polen ha alcanzado recientemente cierta difusión; sin embargo, este producto era prácticamente desconocido como un producto alimenticio durante mucho tiempo, excepto por consumidores vegetarianos o naturistas (10). En la actualidad existen sólo pocos países (por ejemplo, España, China, Hungría, Argentina y Brasil) donde la producción de polen es económicamente significativa; aún así, el mercado de consumo de polen se ha fortalecido durante el último par de décadas (11). Países como Brasil, Argentina, Suiza, España y México han establecido estándares oficiales de calidad y han reconocido al polen como un producto alimenticio (12).

Las características fisicoquímicas del polen dependen de su origen botánico (1). Aunque se pueden encontrar algunos estudios sobre la composición nutricional del polen de abeja en países como Brasil (1), España (4), Australia (13), Argentina (14), Argelia (15), China (16) y Sudáfrica (3), no se ha publicado ningún estudio significativo sobre el polen de abeja colombiano aunque su producción en Colombia ha aumentado constantemente durante la última década debido principalmente a condiciones climáticas excepcionales (17).

El objetivo de esta investigación fue caracterizar el polen de abeja colombiano, proveniente del altiplano cundiboyacense colombiano, desde el punto de vista nutricional y funcional, para contribuir al establecimiento de normas técnicas nacionales y reglamentación oficial, llevando así a establecer un etiquetado sobre la denominación de origen. Los resultados obtenidos son comparados con la normatividad internacional y la bibliografía de referencia, así como con las muestras de polen de abeja comercial de varios países.

\section{MATERIALES Y MÉTODOS}

Tipo de estudio y muestras. Este trabajo de investigación ha sido realizado en el marco del proyecto Estrategias para Establecer la Denominación de Origen de Productos Apícolas en Colombia, financiado por el Ministerio de Agricultura y Desarrollo Rural de Colombia, que contó con la participación de asociaciones de apicultores del altiplano cundiboyacense. En total se recolectaron 196 muestras de polen de abeja (Apis mellifera). Estas muestras se secaron y sus impurezas fueron removidas de la forma como los apicultores normalmente lo hacen ( 2 horas de secado utilizando hornos de aire forzado, con una temperatura inicial del aire menor a $43^{\circ} \mathrm{C}$, seguida por tamizado y un proceso de gradiente de densidad para eliminar las impurezas). Las muestras fueron recolectadas durante los años 2008 y 2011.
Contemporary pollen consumption and marketing has recently achieved certain diffusion; however, this product went practically unrecognized as a food product for a long time, except by vegetarian or naturist consumers (10). At present there are only few countries (i.e. Spain, China, Hungary, Argentina and Brazil) where pollen production is economically significant; even so, the pollen consumer market has strengthened during the last couple of decades (11). Countries such as Brazil, Argentina, Switzerland, Spain and Mexico have established official quality standards and recognized pollen as a food product (12).

Physicochemical characteristics of bee pollen depend on its botanical origin (1). Although some work on the nutritional composition of bee pollen from countries like Brazil (1), Spain (4), Australia (13), Argentina (14), Algeria (15), China (16) and South Africa (3) can be found, no significant study has been published about Colombian bee pollen even though production in Colombia has been constantly increasing over the last decade mainly due to exceptional climatic conditions (17).

The objective of this research was aimed at characterizing Colombian bee pollen, coming from the colombian cundi-boyacense high plateau, from a nutritional and functional point of view, to contribute towards establishing national technical standards and official regulation, thereby leading to establishing origin-denomination labeling. The obtained results are compared to international normativity and referenced bibliography, and also, to samples of commercial bee-pollen from several countries.

\section{MATERIALS AND METHODS}

Type of study and samples. This research work has been made in the context of the project Strategies for establishing Designation of Origin of bee products in Colombia, funded by the Ministry of Agricultural and Rural Development of Colombia, which involved the beekeepers' associations of the Cundiboyacense high plateau. In total, 196 beepollen samples (Apis mellifera) were collected. These samples were dried and impurities were removed as beekeepers usually do (2-hour drying using forced-air ovens, with initial air temperature below $43^{\circ} \mathrm{C}$, followed by sieving and a density-gradient process for removing impurities). The samples were collected during 2008 and 2011. 
Además, nueve muestras de polen comercial de la India, China, Rumania, España, Bulgaria, Hungría y Polonia fueron proporcionadas por fabricantes y comercializadores de polen de los respectivos países durante la exposición del Congreso Mundial de Apicultura 41 - Apimondia, celebrado del 15 al 20 de septiembre de 2009, en Montpellier, Francia.

Todas las muestras fueron llevadas al laboratorio en la presentación comercial habitual donde fueron molidas utilizando un tamiz de $1.5 \mathrm{~mm}$ y conservadas a $4^{\circ} \mathrm{C}$ en la oscuridad hasta que fueran analizadas.

Coordenadas del sitio del estudio. Las coordenadas geográficas del altiplano cundiboyacense colombiana según el IGACORSTOM (18) están relacionadas en la Tabla 1.

\section{Métodos de laboratorio}

pH y acidez. Se mezclaron $2.5 \mathrm{~g}$ de muestra previamente pesado con agua libre de $\mathrm{CO}_{2}$ y se inició la titulación con $0.05 \mathrm{~N}$ de $\mathrm{NaOH}$ hasta un $\mathrm{pH}$ de 8.5. El pH fue calculado utilizando un potenciómetro previamente a la titulación (10).

Humedad. Se pesó una muestra de $3 \mathrm{~g}$ y fue calentada a $65^{\circ} \mathrm{C}$ durante $24 \mathrm{~h}$. El contenido de humedad fue obtenido por la diferencia (10).

Lípidos. Los lípidos se determinaron por el método Soxhlet donde fueron extraídos de la matriz alimentaria mediante arrastre con solvente; este último se separó de los lípidos por calentamiento -AOAC 920.39- (19).

Proteínas. Se determinó siguiendo el método de Kjeldahl con la variación de Winkler. El ácido bórico se utilizó para cuantificar el contenido de nitrógeno en la muestra, aplicando un factor de conversión de N x 6.25 -AOAC 984.13- (19).

Cenizas y minerales. La ceniza fue determinada a través de gravimetría después de incineración en un horno a $600^{\circ} \mathrm{C}$ hasta un peso constante. Los minerales fueron determinados mediante el uso de la ceniza obtenida de esta forma después de las etapas de digestión y dilución. Se utilizó un espectrómetro de absorción atómica AA 240 (Varian Inc., EE.UU.) para tomar mediciones de minerales de acuerdo a las siguientes longitudes de onda: $\mathrm{Na}$ : 599 nm, Mg: 285 nm, K: 766.5 nm, Ca: 422.7 nm, Zn: 213.9 nm y Fe: 248.3 nm -AOAC 968.08- (19).

Fibra dietaria. La fibra dietética se determinó utilizando polen seco y desengrasado, con un tratamiento enzimático de a-amilasa, proteasa y amiloglucosidasa. Una etapa de filtración posterior permite la obtención de fibra dietaria soluble;
In addition, nine samples of commercial beepollen from India, China, Romania, Spain, Bulgaria, Hungary and Poland were provided by manufacturers and marketers houses of pollen from the respective countries during the trade fair of the 41st World Congress of Apiculture Apimondia, September 15th to 20th, 2009, in Montpellier, France.

All samples were brought to laboratory in the usual commercial presentation where they were ground using a $1.5 \mathrm{~mm}$ sieve and kept at $4^{\circ} \mathrm{C}$ in the dark until being analyzed.

Coordinates of the study site. Geographical coordinates of the colombian Cundi-boyacense high plateau according to IGAC-ORSTOM (18) are related in table 1 .

Table 1. List of geographical coordinates of the Cundiboyacense high plateau (18).

\begin{tabular}{ccc}
\hline Location & Latitude $(\mathbf{N})$ & Longitude $(\mathbf{W})$ \\
\hline North-West & $5^{\circ} 55^{\prime} 45.29^{\prime \prime}$ & $72^{\circ} 59^{\prime} 02.26^{\prime \prime}$ \\
North-East & $5^{\circ} 46^{\prime} 45.55^{\prime \prime}$ & $73^{\circ} 42^{\prime} 48.08^{\prime \prime}$ \\
South-West & $4^{\circ} 28^{\prime} 06.53^{\prime \prime}$ & $74^{\circ} 05^{\prime} 16.96^{\prime \prime}$ \\
South-East & $4^{\circ} 52^{\prime} 16.47^{\prime \prime}$ & $74^{\circ} 24^{\prime} 12.10^{\prime \prime}$ \\
\hline
\end{tabular}

\section{Laboratory methods}

pH and acidity. $2.5 \mathrm{~g}$ of previously weighed sample was mixed with $\mathrm{CO}_{2}$-free water and titration was started with $\mathrm{NaOH} 0.05 \mathrm{~N}$ until pH 8.5. $\mathrm{pH}$ is calculated by using a potentiometer previously to titration (10).

Moisture. $3 \mathrm{~g}$ of sample was weighed and heated at $65^{\circ} \mathrm{C}$ for $24 \mathrm{~h}$. Moisture content was obtained by difference $(10)$.

Lipids. Lipids were determined by the Soxhlet method in which they were extracted from the food matrix by solvent drag; this latter is then separated from lipids by heating -AOAC 920.39(19).

Protein. It was determined following the Kjehldahl method with the Winkler variation. Boric acid was used for quantifying nitrogen content in the sample, applying an $\mathrm{N} \times 6.25$ conversion factor-AOAC 984.13-(19).

Ash and minerals. Ash determination was made through gravimetry after incineration in an oven at $600^{\circ} \mathrm{C}$ until constant weight. Minerals were determined by using the ash so obtained after digestion and dilution stages. An AA 240 atomic absorption spectrometer (Varian Inc, USA) was used for taking mineral measurements according 
mientras tanto, la fibra dietaria insoluble se obtiene por precipitación con etanol -AOAC 985.29- $(19,20)$.

Azúcares. Se utilizó HPLC para la evaluación de carbohidratos simples. Se agregaron 40 $\mathrm{ml}$ de agua esterilizada ultrapura a $5 \mathrm{~g}$ de las muestras previamente pesadas para extraer los azúcares. La solución obtenida fue inyectada en el cromatógrafo (sistema de bomba Jasco PU980), mediante el uso de una columna de Varian de $30 \times 0.78 \mathrm{~cm}$ (MetaCarb Ca Plus A5205). Se utilizó agua desgasificada como fase móvil con un flujo de $0.5 \mathrm{ml} / \mathrm{min}$ y la temperatura se fijó en $80^{\circ} \mathrm{C}$ (horno Jasco, CO-2065 Plus). Se usó un detector de índice de refracción (Jasco, RI203 Plus) para la detección del contenido de fructosa, glucosa y sacarosa. Este procedimiento fue adaptado de la metodología propuesta por el método -AOAC 979.23-(19).

Perfil de ácidos grasos. La composición de ácidos grasos se determinó mediante cromatografía de gases, según AOAC 969.33 (19). Los extractos fueron obtenidos mediante el uso de éter de petróleo como solvente y se sometieron a derivatización con metóxido de sodio hasta obtener una emulsión. Los ésteres metílicos producidos fueron medidos mediante cromatografía en un sistema Agilent $7890 \mathrm{~A}$ mediante un columna de $30 \mathrm{~m} \times 0.25 \mathrm{~mm}$ x $0.25 \mu \mathrm{m}$ (OSGE BPX 70). La temperatura del inyector se fijó en $210^{\circ} \mathrm{C}$, el detector temperatura a $220^{\circ} \mathrm{C}$ y la temperatura de la columna se mantuvo a $180^{\circ} \mathrm{C}$. El caudal del gas (He) portador fue de 1 a $1.5 \mathrm{ml} / \mathrm{min}$. La identificación y cuantificación de ácidos grasos se realizó mediante la comparación de los tiempos retención con los estándares que se inyectaron el mismo día.

Análisis estadístico. Las medias y desviaciones estándar fueron calculadas mediante el software Microsoft ${ }^{\circledR}$ Excel.

\section{RESULTADOS}

Las muestras de polen para este estudio fueron tomadas de la región más importante para esta actividad apícola en Colombia, abarcando la mayoría de las variedades disponible en el mercado de este producto en el país. Esta región es el altiplano cundiboyacense que se encuentra localizado a una altitud superior a los 2.500 msnm, en la parte central de los Andes orientales colombianos. Se compone de tierras llanas y altas entre los departamentos de Cundinamarca y Boyacá, con una temperatura media de $15^{\circ} \mathrm{C}$ que puede variar desde 0 hasta $24^{\circ} \mathrm{C}$. Se caracteriza por ser muy fértil para una gran variedad de especies de plantas y tiene temporadas alternantes de floración para varias plantas autóctonas, arbustos y cultivos de importancia económica to the following wavelengths: $\mathrm{Na}: 599 \mathrm{~nm}, \mathrm{Mg}$ : 285nm, K: $766.5 \mathrm{~nm}$, Ca: 422.7 nm, Zn: 213.9 $\mathrm{nm}$ and Fe: $248.3 \mathrm{~nm}$-AOAC 968.08- (19).

Dietary fiber. Dietary fiber was determined by using dried and degreased pollen, with an enzymatic treatment of a-amylase, protease and amyloglucosidase. A posterior filtration stage allows obtaining the soluble dietary fiber; meanwhile, insoluble dietary fiber is obtained by precipitation with ethanol -AOAC 985.29$(19,20)$.

Sugars. HPLC was used for assessing simple carbohydrates. $40 \mathrm{ml}$ of ultrapure sterilized water were added to $5 \mathrm{~g}$ of the previously weighed samples to extract sugars. The obtained solution was injected into the chromatograph (Jasco PU980 pump system), by using a $30 \mathrm{~cm} \times 0.78$ cm Varian column (MetaCarb Ca Plus A5205). Degasified water was used as mobile phase at $0.5 \mathrm{ml} / \mathrm{min}$ flow rate, and temperature was set at $80^{\circ} \mathrm{C}$ (Jasco, CO-2065 Plus oven). A refraction index detector (Jasco, RI-203 Plus) was used for detecting fructose, glucose and sucrose content. This procedure was adapted from the methodology proposed by method -AOAC 979.23- (19).

Fatty acids profile. Fatty acid composition was determined by gas chromatography, according to AOAC 969.33 (19). The extracts were made by using petroleum ether as solvent and underwent derivatization with sodium methoxide until an emulsion was obtained. The methylic esters produced were measured by chromatography in an Agilent 7890A system with a $30 \mathrm{~m} \mathrm{x}$ $0.25 \mathrm{~mm} \times 0.25 \mu \mathrm{m}$ column (OSGE BPX 70). Injector temperature was set at $210^{\circ} \mathrm{C}$, detector temperature was $220^{\circ} \mathrm{C}$ and column temperature was maintained at $180^{\circ} \mathrm{C}$. Carrier gas (He) flow rate was 1 to $1.5 \mathrm{ml} / \mathrm{min}$. Fatty acid identification and quantification was done by comparing retention times with standards injected the same day.

Statistical analysis. Means and standard deviations were calculated by using the software Microsoft ${ }^{\circledR}$ Excel.

\section{RESULTS}

The bee pollen samples for this study were taken from the most important region for this apicultural activity in Colombia, covering most commercially available varieties of this product in the country. This region is the Cundiboyacense high plateau which is located at an altitude higher than 2500 m.a.s.l., on the central part of the colombian eastern Andes. 
durante todo el año. El altiplano cundiboyacense incluye un orobioma de los Andes, caracterizado por la presencia de zonas agrícolas mixtas, pastos para producción lechera, arbustos y vegetación endémica de páramo húmedo en las partes más altas. La mayoría de los apicultores de esta región han formado asociaciones y cooperativas que, junto con el aumento en la demanda, han mejorado esta actividad, estimulando a muchos apicultores a enfocarse exclusivamente en la producción de polen.

En esta región puede encontrarse simultáneamente un gran número de especies de plantas que proveen polen para las abejas Apis mellifera, haciendo que las muestras exhiban una multiplicidad de colores de gránulos y la imposibilidad práctica de tener un polen de abeja monofloral. Aunque en este estudio no se realizó una caracterización palinológica, Nates-Parra et al (21) en un informe anterior, revelaron que Melastomatacea y Fabaceae (incluyendo sus tres subfamilias), Asteracea, Myrtaceae, Euphorbiacea fueron las familias de plantas más importantes que proporcionan polen en el altiplano cundiboyacense.

Para los resultados fisicoquímicos del polen colombiano, se calculó la media y la desviación estándar para todos los datos. En la tabla 2 se presenta la composición promedio del polen de abeja así como los datos de las regulaciones en Brasil, México y Argentina. Por otro lado, en las tablas 3 y 4 se muestran los resultados para la composición promedio de minerales y ácidos grasos, respectivamente.

Table 3. Colombian bee pollen mineral elements (mg/ kg) (Dry basis)

\begin{tabular}{cccccc}
\hline Mineral & Mean & \pm & SD & Minimum & Maximum \\
\hline Sodium & 99.3 & \pm & 79.5 & 8.9 & 206 \\
Potassium & 5.625 & \pm & 1.176 & 3.058 & 7.616 \\
Calcium & 1.717 & \pm & 310 & 1.099 & 2.409 \\
Iron & 70.7 & \pm & 27.8 & 23.2 & 126.6 \\
Magnesium & 1.029 & \pm & 396 & 343 & 1542 \\
Zinc & 47.4 & \pm & 21.6 & 19.8 & 70.6 \\
\hline
\end{tabular}

Por su parte, se analizó el polen comercial de ocho países para realizar una comparación de sus propiedades fisicoquímicas con respecto al producto de las abejas colombianas, que se presentan en la tabla 5 .
Table 2. Chemical analysis of colombian bee-pollen and some international regulatory specifications.

\begin{tabular}{|c|c|c|c|c|c|c|}
\hline \multicolumn{4}{|c|}{ Results } & \multicolumn{3}{|c|}{ Regulatory specifications } \\
\hline & \multicolumn{3}{|c|}{$\mathrm{CCHP}$} & \multirow[b]{2}{*}{ Argentina } & \multirow[b]{2}{*}{ Brazil } & \multirow[b]{2}{*}{ Mexico } \\
\hline & $\begin{array}{l}\text { Mean } \\
\pm \text { SD }\end{array}$ & Min & $\operatorname{Max}$ & & & \\
\hline $\mathrm{pH}$ & $\begin{array}{c}4.6 \\
\pm 0.4\end{array}$ & 3.8 & 5.4 & $\begin{array}{l}\operatorname{Min} 4 \\
\operatorname{Max} 6\end{array}$ & $\begin{array}{l}\operatorname{Min} 4 \\
\operatorname{Max} 6\end{array}$ & Min 4 \\
\hline $\begin{array}{l}\text { Acidity } \\
(\mathrm{meq} / \mathrm{kg})^{*}\end{array}$ & $\begin{array}{l}256 \\
\pm 67\end{array}$ & 155 & 402 & - & $\operatorname{Max} 300$ & - \\
\hline Moisture $(\mathrm{g} / 100 \mathrm{~g})$ & $\begin{array}{c}7.7 \\
\pm 5.2\end{array}$ & 1.8 & 11.8 & Max 8 & $\operatorname{Max} 4$ & $\begin{array}{l}\text { Min } 4.5 \\
\operatorname{Max} 8\end{array}$ \\
\hline Ash $(\mathrm{g} / 100 \mathrm{~g}) *$ & $\begin{array}{l}2.5 \\
\pm 0.4\end{array}$ & 1.5 & 3.2 & Max 4 & $\operatorname{Max} 4$ & $\begin{array}{l}\operatorname{Min} 1.5 \\
\operatorname{Max} 2.2\end{array}$ \\
\hline Lipid $(\mathrm{g} / 100 \mathrm{~g})^{*}$ & $\begin{array}{c}6.9 \\
\pm 3.5\end{array}$ & 2.8 & 9.7 & - & Min 1.8 & $\begin{array}{l}\operatorname{Min} 2.5 \\
\operatorname{Max} 6.5\end{array}$ \\
\hline Protein $(\mathrm{g} / 100 \mathrm{~g}) *$ & $\begin{array}{l}23.8 \\
\pm 3.2\end{array}$ & 16.1 & 32.1 & $\begin{array}{l}\text { Min } 15 \\
\operatorname{Max} 28\end{array}$ & Min 8 & $\begin{array}{l}\operatorname{Min} 12 \\
\operatorname{Max} 18\end{array}$ \\
\hline \multicolumn{7}{|l|}{$\begin{array}{l}\text { Carbohydrates } \\
(\mathrm{g} / 100 \mathrm{~g})^{*}\end{array}$} \\
\hline Fructose & $\begin{array}{l}19.5 \\
\pm 0.9\end{array}$ & 18.1 & 21.3 & - & - & - \\
\hline Glucose & $\begin{array}{l}13.6 \\
\pm 2.4\end{array}$ & 11.6 & 20.3 & - & - & - \\
\hline Sucrose & $\begin{array}{l}6.7 \\
\pm 2.0\end{array}$ & 4.5 & 9.0 & - & - & - \\
\hline $\begin{array}{l}\text { Soluble Dietary } \\
\text { Fiber } \\
(\mathrm{g} / 100 \mathrm{~g})^{*}\end{array}$ & $\begin{array}{c}2.7 \\
\pm 1.8\end{array}$ & 0.5 & 4.6 & - & - & - \\
\hline $\begin{array}{l}\text { Insoluble Dietary } \\
\text { Fiber } \\
(\mathrm{g} / 100 \mathrm{~g})^{*}\end{array}$ & $\begin{array}{l}11.7 \\
\pm 3.3\end{array}$ & 6.5 & 17.6 & - & - & - \\
\hline $\begin{array}{l}\text { Total Dietary Fiber } \\
(\mathrm{g} / 100 \mathrm{~g})^{*}\end{array}$ & $\begin{array}{l}14.5 \\
\pm 3.5\end{array}$ & 7.8 & 18.1 & - & Min. 2 & - \\
\hline
\end{tabular}

$\mathrm{CCHP}=$ Colombia Cundiboyacense High Plateau

*Dry basis. Min: minimum. Max: maximum.

It consists of flat and high lands between the Cundinamarca and Boyacá departments, having a $15^{\circ} \mathrm{C}$ mean temperature which can range from 0 to $24^{\circ} \mathrm{C}$. It is characterized by, being very fertile for a wide variety of plant species and having alternating flowering seasons for several native plants, shrubs and economically important crops throughout the whole year. The Cundiboyacense high plateau has an Andes orobiome, characterized by the presence of mixed agricultural areas, pasture for dairy production, shrub land and damp moor shaving endemic vegetation in the highest parts. Most beekeepers from this region have formed associations and cooperatives, which, together with increasing demand, have enhanced this activity, stimulating many beekeepers to focus exclusively on pollen production.

In this region a large number of plant species can be found simultaneously providing pollen for Apis mellifera bees, causing the samples to display a multiplicity of pellet colors and the practical impossibility of having monofloral bee pollen. Although in this study it was not performed a palynological characterization, Nates-Parra et al (21) in a previous report revealed that Melastomatacea and Fabaceae 
Table 4. Main fatty acids found in Colombian bee pollen (mg/100 mg lipids) (Dry basis).

\begin{tabular}{lccccc}
\hline \multicolumn{1}{c}{ Fatty acid } & Mean & \pm & SD & Minimum & Maximum \\
\hline [C12:0]Lauric & 4.61 & \pm & 2.05 & 0.85 & 9.12 \\
[C14:0]Miristic & 1.33 & \pm & 0.44 & 0.49 & 2.65 \\
[C16:0]Palmitic & 11.33 & \pm & 2.82 & 6.30 & 16.59 \\
[C18:0] Stearic & 1.88 & \pm & 0.41 & 1.07 & 3.04 \\
[C18:1n9c] Oleic & 2.38 & \pm & 0.51 & 1.29 & 3.89 \\
[C18:2n6c] Linoleic & 8.19 & \pm 1.40 & 5.74 & 12.51 \\
[C18:3n3] a-Linolenic & 25.68 & \pm 7.85 & 6.83 & 41.99 \\
[C20:0] Arachidic & 1.27 & \pm 0.32 & 0.63 & 1.54 \\
[C20:3n6c] Homo-y-linolenic & 1.62 & \pm 1.17 & 0.18 & 5.33 \\
(C23:0) Tricosanoic & 1.17 & \pm & 0.60 & 0.24 & 2.26 \\
Saturated Fatty Acids (\%) & 36.62 & \pm 4.92 & 27.31 & 50.79 \\
Unsaturated fatty acids (\%) & 63.38 & \pm & 4.92 & 49.21 & 72.69 \\
Total Fatty acids $(\omega-3)(\%)$ & 37.86 & \pm & 7.43 & 14.97 & 51.26 \\
Total Fatty acids $(\omega-6)(\%)$ & 15.02 & \pm 2.59 & 9.92 & 23.12 \\
Total Fatty acids $(\omega-9)(\%)$ & 8.45 & \pm & 1.58 & 4.87 & 13.08 \\
Unsaturated / Saturated ratio & 1.78 & \pm 0.36 & 0.97 & 2.66 \\
( $\omega-3) /(\omega-6)$ ratio & 2.64 & \pm 0.86 & 0.86 & 4.78 \\
\hline
\end{tabular}

(including its three sub-families), Asteracea, Myrtaceae, Euphorbiacea were the most important plant families providing pollen in the Cundiboyacense high plateau.

For the physicochemical results of colombian bee-pollen, the mean and standard deviation were calculated for all data. Table 2 presents average bee-pollen composition as well as the data of Brazilian, Mexican and Argentinean regulations. On the other hand, tables 3 and 4 show results for average mineral and fatty acid composition, respectively.

On the other hand, commercial bee-pollen from eight countries was analyzed to make a comparison of its physicochemical properties with respect to colombian bee product, which they are presented in table 5 .

Table 5. Average physicochemical composition of commercial bee-pollen samples from several countries.

\begin{tabular}{|c|c|c|c|c|c|c|c|c|c|}
\hline & Hungary & Poland & Slovenia & India & Romania & {$[1]^{S p}$} & ${ }^{n}$ & China & Bulgaria \\
\hline$\overline{\mathrm{pH}}$ & 4.4 & 4.5 & 5.4 & 4.3 & 4.9 & 4.4 & 4.8 & 5.0 & 4.4 \\
\hline Free acidity (meq-kg) & 243 & 332 & 207 & 383 & 237 & 241 & 196 & 351 & 310 \\
\hline Moisture $(\mathrm{g} / 100 \mathrm{~g})$ & 4.9 & 4.0 & 5.9 & 9.1 & 5.1 & 5.2 & 4.4 & 2.0 & 4.6 \\
\hline Ash $(g / 100 \mathrm{~g})^{*}$ & 1.7 & 2.6 & 2.4 & 3.3 & 2.3 & 1.6 & 1.5 & 4.3 & 1.8 \\
\hline Lipids $(\mathrm{g} / 100 \mathrm{~g}) *$ & 4.9 & 5.7 & 5.9 & 8.0 & 4.9 & 5.0 & 5.6 & 5.2 & 5.6 \\
\hline Protein $(\mathrm{g} / 100 \mathrm{~g}) *$ & 16.3 & 25.6 & 21.4 & 26.1 & 22.3 & 20.8 & 15.8 & 17.6 & 19.2 \\
\hline Sodium $(\mathrm{mg} / \mathrm{kg})^{*}$ & 219 & 236 & 240 & 113 & 84 & 379 & 198 & 125 & 199 \\
\hline Potassium $(\mathrm{mg} / \mathrm{kg})^{*}$ & 3607 & 5797 & 5244 & 4794 & 4869 & 3622 & 4487 & 9542 & 4608 \\
\hline Calcium $(\mathrm{mg} / \mathrm{kg})^{*}$ & 1461 & 1654 & 1462 & 2376 & 1657 & 589 & 468 & 1620 & 665 \\
\hline Iron $(\mathrm{mg} / \mathrm{kg})^{*}$ & 40.9 & 56.2 & 42.1 & 197.7 & 89.4 & 57.7 & 28.8 & 63.3 & 47.4 \\
\hline Magnessium $(\mathrm{mg} / \mathrm{kg})^{*}$ & 635 & 1194 & 1135 & 1430 & 865 & 484 & 453 & 2636 & 577 \\
\hline Zinc $(\mathrm{mg} / \mathrm{kg})^{*}$ & 34.3 & 53.2 & 44.3 & 31.3 & 48.7 & 44.3 & 26.1 & 31.4 & 51.9 \\
\hline Insoluble Dietary fiber $(\mathrm{g} / 100 \mathrm{~g}) *$ & 9.6 & 5.7 & - & 12.8 & 8.0 & 8.6 & 8.2 & - & 5.9 \\
\hline Soluble Dietary fiber $(\mathrm{g} / 100 \mathrm{~g}) *$ & 0.8 & 2.3 & - & 1.7 & 0.9 & 3.2 & 1.7 & - & 2.0 \\
\hline Total dietary fiber $(\mathrm{g} / 100 \mathrm{~g}) *$ & 10.5 & 8.1 & - & 14.6 & 9.0 & 11.9 & 10.0 & - & 7.9 \\
\hline
\end{tabular}

* Dry basis.

\section{DISCUSIÓN}

Acidez y pH. El pH del polen de abeja colombiano varió entre 3.8 y 5.4 y presentó un valor promedio general de 4,6 ( \pm 0.4$)$; la acidez osciló entre 155 y $402 \mathrm{~m}_{\mathrm{eq}} / \mathrm{kg}$ con $256( \pm 67) \mathrm{m}_{\mathrm{eq}} / \mathrm{kg}$ como valor medio. Estos dos parámetros fueron correlacionados inversamente, como era de esperarse. A excepción de algunos estudios que generalmente se ocupan de los cambios bioquímicos que el polen sufre dentro de una colmena cuando se convierte en pan de abeja (22), rara vez se ha evaluado la acidez del polen. El pH, por otro lado, ha sido utilizado como indicador de calidad, ya que es un parámetro fácil

\section{DISCUSSION}

Acidity and $\mathbf{p H}$. The $\mathrm{pH}$ of colombian beepollen varied between 3.8 and 5.4 and presented a $4.6( \pm 0.4)$ general mean value; acidity ranged from 155 to $402 \mathrm{~m}_{\mathrm{eq}} / \mathrm{kg}$ with $256( \pm 67) \mathrm{m}_{\mathrm{eq}} / \mathrm{kg}$ as mean value. These two parameters were inversely correlated, as expected. Except for a few works usually dealing with the biochemical changes that pollen suffers inside a bee hive when becoming beebread (22), bee pollen acidity has rarely been assessed. $\mathrm{pH}$, on the other hand, has been used as quality indicator, since it is an easy-to-assess parameter. An elevated acidity 
de evaluar. Una elevada acidez o un bajo $\mathrm{pH}$ puede ser el resultado de actividad microbiana indeseable (23). La regulación argentina establece un intervalo de $\mathrm{pH}$ de 4 a 6, que, según los resultados, parece también ser preciso para el polen colombiano. En comparación con el polen de abeja de otros países que se muestra en la tabla 5, se puede encontrar que los valores de $\mathrm{pH}$ y acidez son similares al polen del colombiano. En particular, la acidez del polen de Polonia, India, China y Bulgaria son superiores a $300 \mathrm{meq} / \mathrm{kg}$ y ésos no satisfarían la normatividad brasileña.

Humedad. Los apicultores reducen el contenido de humedad en el polen mediante secado (a partir de los valores iniciales que oscilan entre el $18 \%$ y el 34\% en el altiplano cundiboyacense) (10) para reducir la actividad acuosa y así evitar el deterioro microbiano. El polen de abeja colombiano varía de 1.8 a $11.8 \mathrm{~g} / 100 \mathrm{~g}$ en el presente estudio y presentó un valor medio de $7.7( \pm 5.2) \mathrm{g} / 100 \mathrm{~g}$. La mayoría de las muestras (93\%) cumplían con la norma argentina del $8 \%$ como contenido máximo permisible de humedad pero sólo el $19 \%$ de las mismas cumplían con las regulaciones brasileñas que indican un máximo de $4 \%$. El polen de abeja menor aproximadamente $\sim 6 \mathrm{~g} / 100 \mathrm{~g}$ se encuentra en el rango aceptable para la estabilidad de almacenamiento, teniendo en cuenta que un contenido de humedad inferior al $4 \%$ puede también inducir reacciones de rancidez (11). El contenido de humedad del polen de abeja internacional osciló entre 2.0 (China) y 9.1 (India); es evidente que las técnicas de secado de polen han sido pobremente estudiadas y estandarizadas, razón por la cual es posible encontrar diferencias considerables en el contenido acuoso del polen de abeja en todo el mundo.

Proteína. El contenido de proteína en el polen de abeja es una de sus características nutricionales más importante; el valor del mismo osciló entre 16.1 y $32.1 \mathrm{~g} / 100 \mathrm{~g}$ (base seca) evaluado mediante un factor $\mathrm{N} \times 6.25$ con una media de $23.8( \pm 3.2)$ $\mathrm{g} / 100 \mathrm{~g}$. Estos resultados fueron similares a los obtenidos por Szczesna (24) para polen proveniente de Polonia, por Estevinho et al (25) para el polen recolectado en Portugal y por Almeida-Muradian et al (1) para el polen brasileño. Hubo diferencias en el contenido proteínico del polen de España según lo reportado por González-Martín et al (4), donde fue menor; las diferencias encontradas en la cantidad total de proteína fueron causadas por su origen botánico (26). Las regulaciones brasileñas sobre el contenido de proteína del polen de abeja establecen un valor mínimo del $8 \%$ (peso seco) mientras que las regulaciones argentinas establecen un rango del $15 \%$ al $28 \%$ (peso seco); todas las muestras del altiplano cundiboyacense analizadas estuvieron por encima del mínimos para los valores anteriores y el $11 \%$ de las mismas fueron superiores al estándar or low $\mathrm{pH}$ can result from undesirable microbial activity (23). Argentinean regulation establishes a $\mathrm{pH}$ range from 4 to 6 , which, according to the results, seems to be accurate for colombian pollen as well. In comparison with bee-pollen from other countries, shown in table 5 , it can be found that $\mathrm{pH}$ and acidity values are similar to colombian pollen. Particularly, acidity of Poland, Indian, Chinese and Bulgarian bee-pollens are higher than $300 \mathrm{meq} / \mathrm{kg}$, and those would not fulfill the brazilian normativity.

Moisture. Beekeepers reduce bee pollen moisture content by drying (from initial values ranging from $18 \%$ to $34 \%$ on the Cundiboyacense high plateau) (10) to reduce water activity and so prevent microbial deterioration. Colombian bee-pollen varied from 1.8 to $11.8 \mathrm{~g} / 100 \mathrm{~g}$ in the present study and had 7.7 ( \pm 5.2$) \mathrm{g} / 100 \mathrm{~g}$ mean value. Most samples (93\%) met the argentinean standard of $8 \%$ as maximum allowable moisture content but only $19 \%$ of them complied with brazilian regulations stating a maximum of $4 \%$. Bee pollen below $\sim 6 \mathrm{~g} / 100 \mathrm{~g}$ is in the acceptable range for storage stability, having into account that also a moisture content lower than $4 \%$ may induce to rancidity reactions (11). Moisture content of international bee-pollen ranged from 2.0 (China) to 9.1 (India); it is clear that bee-pollen drying techniques are poorly studied and standardized, that is why it can be found considerable differences in the water content of bee-pollen worldwide.

Protein. Bee-pollen protein content is one of its most regarded nutritional features; this ranged from 16.1 to $32.1 \mathrm{~g} / 100 \mathrm{~g}$ (dry basis) evaluated by using factor $\mathrm{N} \times 6.25$, having a $23.8( \pm 3.2) \mathrm{g} / 100 \mathrm{gmean}$. These results were similar to those obtained by Szczesna (24) for pollen from Poland, by Estevinho et al (25) for pollen collected in Portugal and by AlmeidaMuradian et al (1) for brazilian pollen. There were differences with spanish pollen protein content as reported by González-Martín et al (4), where it was lower; the differences found in the total amount of protein were due to their botanical origin (26). Brazilian regulations for bee pollen protein content set an $8 \%$ minimum (dry weight) while argentinean regulations state a range from $15 \%$ to $28 \%$ (dry weight); all Cundiboyacense high plateau samples analyzed were above the minimum for both the foregoing and $11 \%$ of them were above the maximum argentinean standard. In comparison to international bee-pollen, colombian had one of the greatest proteins content, similar to bee-pollen from Romania $(22.3 \mathrm{~g} / 100 \mathrm{~g})$ and only below of Poland (25.6 $\mathrm{g} / 100 \mathrm{~g})$ and India $(26.1 \mathrm{~g} / 100 \mathrm{~g})$. 
máximo de Argentina. En comparación con el polen internacional, Colombia tuvo uno de los mayores contenidos de proteínas, similar al polen de abeja de Rumania ( $22.3 \mathrm{~g} / 100 \mathrm{~g}$ ) y sólo por debajo de Polonia $(25.6 \mathrm{~g} / 100 \mathrm{~g})$ e India $(26.1 \mathrm{~g} / 100 \mathrm{~g})$.

Fibra. El IDF varió entre 6.5 y $17.6 \mathrm{~g} / 100 \mathrm{~g}$, con una media de $11.7( \pm 3.3) \mathrm{g} / 100 \mathrm{~g}$. Por otro lado, el SDF varió de 0.5 a $4.6 \mathrm{~g} / 100 \mathrm{~g}$, con un valor medio de $2.7( \pm 1.8) \mathrm{g} / 100 \mathrm{~g}$ (base seca). La cantidad de SDF fue considerablemente más alta en comparación con la mayoría de productos manufacturados. La media de fibra dietaria total fue $14.5 \mathrm{~g} / 100 \mathrm{~g}$. Entre el polen internacional analizado, India tuvo el mayor contenido de IDF $(12.8 \mathrm{~g} / 100 \mathrm{~g})$, mientras que otros productos internacionales analizados oscilaron entre 5.7 y 9.6. Una muestra de España tuvo el mayor contenido de SDF $(3.2 \mathrm{~g} / 100 \mathrm{~g})$, un valor notablemente alto en comparación con otros países (rangos de 0.8 a $2.3 \mathrm{~g} / 100 \mathrm{~g}$ ). Es posible observar que la proporción de fibra dietaria soluble e insoluble del polen colombiano constituye otro factor de funcionalidad, con un contenido más alto incluso que productos similares de otras regiones.

Carbohidratos. Se identificaron y cuantificaron tres azúcares principales; fructosa fue el más abundante (19.45 $\pm 0.92 \mathrm{~g} / 100 \mathrm{~g}$ peso seco), seguido de glucosa $(13.58 \pm 2.35 \mathrm{~g} / 100 \mathrm{~g})$ y sacarosa $(6.68 \pm 2.03 \mathrm{~g} / 100 \mathrm{~g})$. El coeficiente F/G varió entre 1.20 y 1.61. La normatividad vigente en los distintos países no considera límites mínimos o máximos para el contenido de carbohidratos en el polen; sin embargo, en comparación con los datos obtenidos de Qian et al (5), para el polen de abeja chino, los resultados difieren notablemente, especialmente en relación con la concentración de sacarosa que ha sido reportada como considerablemente más alta.

Lípidos. El contenido total de lípidos se mostró altamente variable y osciló entre 2.8 y $9.7 \mathrm{~g} / 100$ $\mathrm{g}$ (media de $6.9( \pm 3.5) \mathrm{g} / 100 \mathrm{~g})$. El polen de esta región cumplió con el mínimo de 1.8 (peso seco) de lípidos totales especificado en las regulaciones brasileñas y mexicanas. El contenido de lípidos es altamente variable y depende de la cantidad de ácidos grasos, carotenos y vitaminas presentes en el polen. Los valores medios fueron similares a aquellos encontrados en variedades de polen de otros países, que oscilaron de 4.9 a $8.0 \mathrm{~g} / 100$ $\mathrm{g}$, siendo el polen de la India aquel con el mayor contenido. No obstante, es importante señalar que la desviación estándar apreciable obtenida para el polen de abeja colombiano es causada por la diversidad del origen botánico de los pólenes de esta región.

Perfil de ácidos grasos. El perfil de ácidos grasos fue analizado para el polen del altiplano
Fiber. IDF varied from 6.5 to $17.6 \mathrm{~g} / 100 \mathrm{~g}$, having an $11.7( \pm 3.3) \mathrm{g} / 100 \mathrm{~g}$ mean. On the other hand SDF varied from 0.5 to $4.6 \mathrm{~g} / 100 \mathrm{~g}$, having a 2.7 ( \pm 1.8$) \mathrm{g} / 100 \mathrm{~g}$ mean (dry basis). The amount of SDF was considerably high compared to most manufactured products. The mean for total dietary fiber was of $14.5 \mathrm{~g} / 100 \mathrm{~g}$. Among analyzed international bee-pollen, India had the greatest IDF content $(12.8 \mathrm{~g} / 100 \mathrm{~g})$, meanwhile other analyzed international products ranged from 5.7 to 9.6. One sample from Spain had the greatest content of SDF $(3.2 \mathrm{~g} / 100 \mathrm{~g})$, a notorious high value compared to other countries (ranges from 0.8 to $2.3 \mathrm{~g} / 100 \mathrm{~g}$ ). It can be seen that the proportion of soluble and insoluble dietary fiber of colombian bee-pollen constitutes another functionality factor, having even higher contents than similar products from other regions.

Carbohydrates. Three main sugars were identified and quantified; fructose was the most abundant (19.45 $\pm 0.92 \mathrm{~g} / 100 \mathrm{~g}$ dry weight), followed by glucose $(13.58 \pm 2.35 \mathrm{~g} / 100 \mathrm{~g})$ and sucrose $(6.68 \pm 2.03 \mathrm{~g} / 100 \mathrm{~g}) . \mathrm{F} / \mathrm{G}$ ratio varied between 1.20 and 1.61 . The existing regulation in different countries does not consider minimum or maximum limits for carbohydrate content in pollen; however, in comparison to data obtained for Qian et al (5), for chinese bee pollen, results differ noticeably, especially regarding sucrose concentration which has been reported as being considerably higher.

Lipids. Total lipid content was highly variable and ranged from 2.8 to $9.7 \mathrm{~g} / 100 \mathrm{~g}$ (mean 6.9 $( \pm 3.5) \mathrm{g} / 100 \mathrm{~g})$. Pollens from this region met the minimum $1.8 \%$ (dry weight) total lipids specified in brazilian and mexican regulations. The content of lipids is highly variable and dependent on the amount of fatty acids, carotenes and vitamins present in pollen. Mean values were similar to those found in pollen varieties from other countries, which ranged from 4.9 to $8.0 \mathrm{~g} / 100 \mathrm{~g}$, with pollen from India having the highest content. Nevertheless, it is important to note that the appreciable standard deviation obtained for colombian bee-pollen is due to the diversity of botanical origin of pollens from this region.

Fatty acid profile. The fatty acid profile was analyzed for pollens from Cundiboyacense highplateau. a-linolenic [C18:3n3] was found to be the most important fatty acid $(25.68 \mathrm{mg} / 100 \mathrm{mg}$ mean lipid concentration equal to $39.2 \%$ of fatty acids found) followed by palmitic [C16:0] $(11.33 \mathrm{mg} / 100 \mathrm{mg}$ of lipids), linoleic [C18:2n6c] $(8.19 \mathrm{mg} / 100 \mathrm{mg}$ of lipids), lauric [C12:0] $(4.61 \mathrm{mg} / 100 \mathrm{mg}$ of lipids), oleic [C18:1n9c] (2.38 mg/100 mg of lipids), stearic 
cundiboyacense. Se encontró que el ácido a-linolénico [C18:3n3] fue el ácido graso más importante (la concentración media de lípidos de $25.68 \mathrm{mg} / 100 \mathrm{mg}$ fue equivalente al $39.2 \%$ de los ácidos grasos encontrados) seguido por el ácido palmítico [C16:0] (11.33 mg/100 mg de lípidos), linoleico [C18:2n6c] (8.19 mg/100 mg de lípidos), láurico [C12:0] (4.61 mg/100 mg de lípidos), oleico [C18:1n9c] (2.38 mg/100 mg de lípidos), esteárico [C18:0], cis-8,11,14-eicosatrienoico [C20:3n6], mirístico [C14:0] y tricosanoico [C23:0]. La proporción de ácidos grasos insaturados/saturados fue 1.78; se han encontrado previamente proporciones mayores a 1 en polen de abeja $(3,6,25)$, con las variaciones siendo causadas por el origen botánico. La fracción lipídica también puede contener otros ácidos grasos, pigmentos, ceras, esteroles, vitaminas liposolubles, alcoholes pesados e hidrocarburos (3).

Cenizas y minerales. El contenido medio de cenizas para el polen de abeja colombiano fue 2.5 $( \pm 0.4) \mathrm{g} / 100 \mathrm{~g}$ (base seca) que varía desde $1.5 \mathrm{a}$ $3.2 \mathrm{~g} / 100 \mathrm{~g}$. Estos resultados son consistentes con los resultados anteriores $(1,13)$; todas las muestras cumplieron con las normas argentinas y brasileñas que establecen un máximo del 4\% (peso seco). En una comparación con polen de abeja internacional se obtuvieron valores similares para el contenido de cenizas, el cual oscila entre 1.5 y $3.3 \mathrm{~g} / 100 \mathrm{~g}$, con excepción de China, con $4.3 \mathrm{~g} / 100 \mathrm{~g}$.

Por otro lado, se evaluaron seis minerales (Tabla 3 ), siendo el potasio el más concentrado, seguido en orden decreciente por calcio, magnesio, sodio, hierro y zinc. Los resultados fueron consistentes con otros resultados $(1,13)$. Este perfil mineral respalda la idea de que el polen es un suplemento nutricional. El polen de abeja de otros países ha mostrado una gran diversidad; con el contenido de potasio y magnesio variando entre 3.607 y $9.542 \mathrm{mg} / \mathrm{kg}$ y de 453 a $2.636 \mathrm{mg} / \mathrm{kg}$, respectivamente, siendo China el país con el mayor contenido en ambos casos; esto explicaría el alto valor inusual para el contenido de cenizas. Para el calcio y el hierro, los valores oscilaron entre 468 y $2.376 \mathrm{mg} / \mathrm{kg}$ y de 28.8 a $197.7 \mathrm{mg} / \mathrm{kg}$, respectivamente, teniendo la India el mayor contenido en ambos casos. Para sodio y zinc, los valores obtenidos no han tenido una variabilidad considerable entre países, que varía desde 84 a $379 \mathrm{mg} / \mathrm{kg}$ y de 26.1 a $53.2 \mathrm{mg} /$ $\mathrm{kg}$, respectivamente. Particularmente, el polen de Colombia mostró un valor considerablemente alto para el contenido de potasio, calcio y hierro y también, un bajo valor de sodio.

Como conclusiones, las características fisicoquímicas y funcionales del polen del altiplano cundiboyacense podrían desempeñar un papel importante para controlar la calidad de este producto y cumplirían los requerimientos internacionales, a excepción del
[C18:0], cis-8,11,14-eicosatrienoic [C20:3n6], miristic [C14:0] and tricosanoic [C23:0]. The unsaturated/saturated fatty acid ratio was 1.78 ; ratios greater than 1 have been previously found in bee pollen $(3,6,25)$, variations being due to botanical origin. The lipid fraction may also contain other fatty acids, pigments, waxes, sterols, liposoluble vitamins, heavy alcohols and hydrocarbons (3).

Ash and minerals. Mean ash content found for colombian bee-pollen was $2.5( \pm 0.4) \mathrm{g} / 100 \mathrm{~g}$ (dry basis) ranging from 1.5 to $3.2 \mathrm{~g} / 100 \mathrm{~g}$. These results agreed with previous findings $(1,13)$; all samples met both argentinean and brazilian standards establishing a maximum $4 \%$ (dry weight). A comparison with international bee-pollen showed similar values for ash content ranging between 1.5 and $3.3 \mathrm{~g} / 100 \mathrm{~g}$, except for China with $4.3 \mathrm{~g} / 100 \mathrm{~g}$.

On the other hand, six minerals were assessed (Table 3 ), potassium being the most concentrated, followed in decreasing order by calcium, magnesium, sodium, iron and zinc. The results agreed with other findings $(1,13)$. Such mineral profile supports the idea of pollen being a nutritional supplement. Beepollen from other countries has shown a great diversity; potassium and magnesium content ranged from 3607 to $9542 \mathrm{mg} / \mathrm{kg}$ and 453 to $2636 \mathrm{mg} / \mathrm{kg}$, respectively, with China having the highest content in both cases; this would explain the unusual high value for ash content. In calcium and iron, values ranged from 468 to 2376 $\mathrm{mg} / \mathrm{kg}$ and 28.8 to $197.7 \mathrm{mg} / \mathrm{kg}$, respectively, with India having the highest content in both cases. For sodium and zinc, the obtained values have not had a considerable variability among countries, ranging from 84 to $379 \mathrm{mg} / \mathrm{kg}$ and 26.1 to $53.2 \mathrm{mg} / \mathrm{kg}$, respectively. Particularly, Colombian bee-pollen showed a considerable high value for potassium, calcium and iron content, and also, a low value for sodium.

As conclusions, physicochemical and functional characteristics of pollens from the CundiBoyacense high plateau could play an important role for quality control of this product and would satisfy international requirements, except for moisture content, where it is necessary to improve drying conditions to obtain a more suitable product. The particularities of colombian pollen composition demand that local standards become defined; the data presented here would thus provide a suitable basis for such work.

Particularly, high levels of minerals were found in analysis compared to several reports and analyzed bee-pollen from several countries. 
contenido de humedad, ya que resulta necesario mejorar las condiciones de secado para obtener un mejor producto. Las particularidades de la composición del polen colombiano requieren de la definición de normas locales; los datos presentados aquí proporcionarían por lo tanto una base adecuada para dicho estudio.

En particular, se han encontrado altos niveles de minerales en el análisis en comparación con varios informes y el polen analizado de varios países. Los altos niveles de potasio, proteína, fibra dietaria y lípidos indican la posibilidad de utilizar el polen como un suplemento dietario. Se recomienda realizar un análisis posterior centrado en los componentes y propiedades bio-activas y la caracterización de la fracción volátil y características sensoriales para caracterizar totalmente el polen colombiano y así diferenciarlo localmente y de otras variedades mundiales. Se espera que este trabajo constituya una herramienta importante para catalogar y reconocer al polen colombiano como una fuente beneficiosa de nutrientes naturales.

\section{Agradecimientos}

Los autores agradecen al Ministerio de Agricultura y Desarrollo Rural de Colombia, el Departamento Administrativo de Ciencia, Tecnología e Innovación de Colombia (COLCIENCIAS) y las siguientes asociaciones de apicultura: Asociación de Apicultores de Boyacá (ASOAPIBOY), la Asociación de Apicultores de la Región del Sumapaz (ASOAPIS), la Asociación de Apicultores Conservacionistas de la Sierra Nevada de Santa Marta (APISIERRA), la Asociación Apícola Comunera (ASOAPICOM), Apicultura Los Cerezos y Apiarios Los Cítricos.
The high potassium, protein, dietary fiber and lipids levels indicated the possibility of using pollen as a dietary supplement. Further analysis focused on bioactive components and properties and characterizing the volatile fraction and sensory characteristics are highly recommended for fully characterizing colombian bee pollens and differentiating them locally and from other varieties from around the world. It is expected that this work would be an important tool for cataloguing and recognizing colombian pollen as being a beneficial source of natural nutrients.

\section{Acknowledgments}

The authors thank the colombian Ministry of Agriculture and Rural Development, the colombian Science, Technology and Innovation Department (COLCIENCIAS), and the following beekeeping associations: Asociación de Apicultores de Boyacá (ASOAPIBOY), the Asociación de Apicultores de la región del Sumapaz (ASOAPIS), the Asociación de Apicultores Conservacionistas de la Sierra Nevada de Santa Marta (APISIERRA), the Asociación Apícola Comunera (ASOAPICOM), Apicultura Los Cerezos and Apiarios Los Cítricos.

\section{REFERENCES}

1. Almeida-Muradian L, Pamplona L, Coimbra S, Barth O. Chemical composition and botanical evaluation of dried bee pollen pellets. J Food Compos Anal 2005; 18(1):105-111.

2. Del Risco C, Pérez A, Álvarez V, Rodríguez G, Leiva V, Puig Y, et al. Bacterias ácido-lácticas para ensilar polen apícola. Revista CENIC. Ciencias Biológicas 2012; 43(1):17-21.

3. Human $\mathrm{H}$, Nicolson S. Nutritional content of fresh, bee-collected and stored pollen of Aloe geatheadii va. davyana (Asphodelaceae). Phytochemistry 2006; 67:1486-1492.
4. Gonzalez-Martin I, Hernandez-Hierro J, Barros-Ferreiro N, Cordon C, GarciaVillanova R. Use of NIRS technology with a remote reflectance fibre-optic probe for predicting major components in bee pollen. Talanta 2007; 72:998-1003.

5. Qian WL, Khan Z, Watson DG, Fearnley J. Analysis of sugars in bee pollen and propolis by ligand exchange chromatography in combination with pulsed amperometric detection and mass spectrometry. J Food Compos Anal 2008; 21(1):78-83. 
6. Xu X, Dong J, Mu X, Sun L. Supercritical $\mathrm{CO} 2$ extraction of oil, carotenoids, squalene and sterols from lotus (Nelumbo nucífera Gaertn) bee pollen. Food Bioprod Process $2011 ; 89(1): 47-52$.

7. Xu L, Sun I, Dong L, Zhang H. Breaking the cells of rape bee pollen and consecutive extraction of functional oil with supercritical carbon dioxide. Innovat Food Sci Emerg Tech 2009; 10:42-46.

8. Pinto $B$, Caciagli $F$, Riccio E, Reali D, Šarić A, Balog T, et al. Antiestrogenic and antigenotoxic activity of bee pollen from Cystus incanus and Salix alba as evaluated by the yeast estrogen screen and the micronucleus assay in human lymphocytes. Eur J Med Chem 2010; 45(9):4122-4128.

9. Mărghitaş LA, Stanciu OG, Dezmirean DS, Bobiş O, Popescu O, Bogdanov S, et al. In vitro antioxidant capacity of honeybeecollected pollen of selected floral origin harvested from Romania. Food Chem 2009; 115(3):878-883.

10. Diaz C, Zuluaga C, Fuenmayor C, Martinez T. Special features of pollen production in Colombia. 41st World Congress of Apiculture. Montpellier, France; Apimondia: 2009.

11. Campos M, Bogdanov S, Almeida-Muradian L, Szczesna T, Mancebo Y, Frigerio C, et al. Pollen composition and standardisation of analytical methods. J Apicult Res 2008; 47(2): 156-163.

12. Bogdanov S. The Bee Pollen Book. Bulgaria: Bee Product Science; 2011.

13. Somerville D, Nicol H. Mineral content of honeybee-collected pollen from sothern New South Wales. Aust J Exp Agr 2002; 42(8): 1131-1136.

14. Baldi B. Composición bromatológica del polen argentino. Ciencia, docencia y tecnología. Argentina: Universidad Nacional de Entre Ríos; 2004.

15. Rebiai A, Lanez T. Chemical composition and antioxidant activity of Apis mellifera bee pollen from northwest Algeria. J Fund Appl Sci 2012; 4(2):26-35.

16. Yang K, Wu D, Ye X, Liu D, Chen J, Sun P. Characterization of Chemical Composition of Bee Pollen in China. J Agr Food Chem 2013; $61: 708-718$.
17. Martínez T. Diagnóstico de la actividad apícola y de la crianza de abejas en Colombia. Colombia: Ministerio de Agricultura y Desarrollo Rural - Dirección de Cadenas Productivas. Instituto Intermaericano de Cooperación para la Agricultura; 2006.

18. IGAC-ORSTOM. Estudio regional integrado del Altiplano Cundiboyacense: estudio general de suelos. Bogotá, Colombia; 1984.

19. AOAC. Official Methods of Analysis of AOAC International. Association Official Analytical Chemists; 2005.

20. Díaz C, Zuluaga C, Morales C, Quicazán M. Determinación de fibra dietaría en polen apícola colombiano. Vitae 2012; 19(S1):454-456.

21. Nates-Parra G, Montoya P, Obregón D, Ramírez N, Solarte V, Chamorro F. La melisopalinología como herramienta para la diferenciación por origen botánico de productos de abejas en Colombia. Sincelejo, Colombia: Encuentro Nacional de Apicultura; 2010.

22. Čeksterytè V, Račys J, Kaškonienè V, Venskutonis P. Fatty acid composition in beebread. Biologija 2008; 54(4):253-257.

23. Fuenmayor C, Quicazán M, Torres A, Rubio A. A beebread-inspired functional dietary supplement by means of solid state fermentation of honeybee-collected pollen with probiotic. Montpellier, France: Apimondia; 2009.

24. Szczesna T. Protein content and amino acid composition of bee-collected pollen from selected botanical origins. J Apic Sci 2006; 50(2):81-90.

25. Estevinho LM, Rodrigues S, Pereira AP, Feás $X$. Portuguese bee pollen: palynological study, nutritional and microbiological evaluation. Int J Food Sci Technol 2012; 47(2):429-435.

26. Almaraz-Abarca N, Campos M, Ávila-Reyes J, Naranjo-Jiménez N, Herrera-Corral J, González-Valdez L. Variability of antioxidant activity among honeybee-collected pollen of different botanical origin. Interciencia 2004; 29(10): 1-12. 